\title{
Let's work together
}

Schoon, I. (2015). Let's work together. Towards Interdisciplinary Collaboration. Research in Human Development, 12: 350-355

\section{Let's work together}

Ingrid Schoon

University College London, Institute of Education

I.Schoon@ioe.ac.uk

Correspondence concerning this article should be addressed to Ingrid Schoon, University College London, Institute of Education, 20 Bedford Way, London WC1H 0AL, tel: +44 2076126238 (direct), email: I.Schoon@ioe.ac.uk

15 May 2015 


\title{
Let's work together
}

\begin{abstract}
Key issues in the study of human development, such as minimizing the impact of poverty and deprivation and improving health, wellbeing and attainment for all, require a collective effort by different disciplines. Research and funding agencies around the world have recognised the need for inter-disciplinary research (IDR) in solving important problems and accelerating scientific discovery. Yet there are persisting barriers and challenges to interdisciplinary collaboration and research including institutional structures and training, the organisation and funding of research, publication culture and assessment criteria. This paper discusses the strengths and limitations of doing IDR, advocating a collaborative approach and associated institutional as well as individual level change.
\end{abstract}

Keywords: Interdisciplinary Research 


\section{Let's work together}

Let's work together

Key issues in the study of human development, such as minimizing the impact of poverty and deprivation and improving health, wellbeing and attainment for all, require a collective effort by different disciplines. Research and funding agencies around the world have recognised the need for inter-disciplinary research (IDR) in solving important problems and accelerating scientific discovery. For example, The National Academy of Sciences (2005) defines IDR as one of the most productive and inspiring of human pursuits, facilitating conversations and connections that lead to new knowledge. Psychologists, sociologists, and town planners might combine their knowledge, research tools, and technologies to more robustly address and tackle complex issues of human development such as mental health problems, crime and drug use of urban populations. By engaging seemingly unrelated disciplines, established gaps in terminology, approach, and methodology might gradually be reduced. Yet the pursuit of IDR in the study of human development is constrained due to a number of reasons, including disciplinary structures of universities, organization and funding of research, publication culture and the peer review process as well as individual factors and interests. My wish is for dialog and debate between different disciplines so that there can be more convergence and collaboration and a better understanding of human development over time and in context. Moreover, barriers to IDR should be removed to enable a move towards more integrated research on crucial issues of human development in context.

\section{Human Development in Context: It is complex}

Human development does not occur in a social vacuum. It is not possible to understand behaviour development of school-aged children without taking into account the role of parents, peers, teachers, school characteristics, neighbourhoods and the wider socio- 


\section{Let's work together}

economic context. Immense strides have been made towards gaining a better understanding of the multiple and interlinked factors and processes shaping human development, yet even regarding key terms such as 'development ' there are differences in definition across disciplines, reflecting different paradigms of inquiry and approach. For example, in psychology the term development refers to regularities in ontogenetic changes, while in sociology it is used to describe structural change or temporal norms and expectations related to age and social transitions. Despite differences regarding the explanation and primary focus on either the individual or the social structure, there are also many commonalities in assumptions. For instance, the recognition that human development a.) takes time and that it reflects cumulative experiences (e.g. the accumulation of individual resources such as educational credentials or capabilities); b.) that it is embedded in a wider social context and involves interactions with significant others in direct proximity (for example with parents, peers, teachers) as well as with the wider macro-social context (i.e. institutional regulations, welfare systems or economic performance of one's country); c. that developmental tasks, such as the transition into and through education or into the labour market are shaped by individual choice, which in turn is informed through negotiations with significant others, as well as institutional structures and opportunities. For example, decisions about subject choice or whether to participate in higher education are influenced by previous levels of attainment; own interests, self concepts and motivations; experiences in the school system; parental support; economic circumstances; institutional regulations and support regarding transition processes.

Bringing together evidence and approaches from different perspectives can help to advance critical thinking, uncovering unquestioned pre-conceptions and potential bias, and encourage tolerance of uncertainty as well as ability of working in teams. For example, the 


\section{Let's work together}

parable of the blind men and the elephant, originating in ancient Indian texts, addresses the manifold nature of truth. Six blind men were asked by a king to determine what an elephant looked like. One blind man feels the elephant's leg and says the elephant is like a pillar; another one feels the tail and says the elephant is like a rope; one who feels the trunk says the elephant is like a tree branch; the one who feels the ear says the elephant is like a hand fan; the one who feels the belly says the elephant is like a wall; and the one who feels the tusk says the elephant is like a solid pipe. The king then explains to them: All of you are right. The reason every one of you is telling it differently is because each one of you touched the different part of the elephant. So, actually the elephant has all the features you mentioned.

Removing the blind folds and barriers to potential inter-disciplinary collaboration will help to broaden the range of investigation, bringing with it new insights, new technologies, and new ways of seeing. It might even contribute to the development of new hybrid disciplines that are conceptually and methodologically more sophisticated, especially regarding the study of societal problems and their resolution. Knowledge extension and innovation frequently take place at the interface of disciplines. Facilitating cross fertilisation across research units will allow staff and students to adopt a wider, more comprehensive perspective on issues of mutual interest, and open up opportunities for debate and discussion and the development of exciting and new research projects. This in turn will help to create a new generation of students who will be able to combine expertise in one discipline, understanding its rigour and depth, with the capacity to reach out to other disciplines and work in interdisciplinary teams.

\section{What is IDR?}

IDR is a process of addressing a topic or problem that is too broad or complex to be dealt with by a single discipline or profession and draws on different disciplinary 


\section{Let's work together}

perspectives to integrate their insights to create a more comprehensive understanding (Klein, 1990). There is however considerable ambiguity in how the term is defined (Frodeman, Klein \& Mitcham, 2009; Jacobs \& Frickel, 2009). The scope of interdisciplinarity can vary from narrow to middle-ranged or horizontal forms of interdisciplinarity among neighbouring disciplines with compatible epistemologies (for example between cognitive psychology and neuropsychology) to broadly determined, vertical, and grand-scale forms among disciplines with more divergent epistemologies (Klein, 2008).

A first step in doing IDR is to define its scope and purpose, and to justify why an interdisciplinary approach is needed (Repko, 2008). The problem should be of societal relevance and has to be stated clearly and concisely. It has to be of interest and relevant to two or more disciplines, who can offer important insights into the problem at hand, but have not yet addressed the problem comprehensively. Adopting an interdisciplinary approach should provide a better understanding of the historical, cultural, and social background of the problem, its varied contexts, and show paths and insights of prior disciplinary research.

IDR demands a serious engagement with multiple disciplines, yet does not undermine disciplinary identity and knowledge. The researchers who conduct inter-disciplinary research should be excellent specialists of a discipline (OECD, 1998), who are recognised as leaders in their field, who are prepared to engage in interdisciplinary translation and to develop a 'synthesising mind' (Gardner, 2006). IDR requires depth as well as breadth of experience. Effective interdisciplinary research demands a willingness to engage with the terminology, perspective, theory, and method of different disciplinary components of the research programme, to work out how they relate to one another and how they should be combined to deliver an overall integrated outcome. 


\section{Let's work together}

IDR is based on the recognition that each discipline has its own epistemology, concepts, theories, and methods. It has to identify conflicts between disciplinary based insights, locate their sources, and create a common ground, 'a bridge' to span the disciplinary chasm and to bring out potential communalities (Repko, 2008). The process of identifying a common ground can depend on the motivation of researchers to engage in collaboration, the influence of group dynamics and individual contributions, and most importantly the effective communication in inter-disciplinary teams. It becomes necessary to transcend existing knowledge traditions and disciplinary jargon, and a number of tools have been identified to support the collaboration process, including the use of story-lines and metaphor, choice of vocabulary, the nature of dialogue and the role of mediating agents (Jeffrey, 2003).

\section{Challenges}

A variety of factors can contribute to the success or failure of IDR, including assessment criteria used regarding publications and the peer review process, individual characteristics and preferences, as well as the disciplinary structure of universities and the organisation and funding of research (Feller, 2002; Borrego, Boden \& Newswander, 2014).

Research assessment. IDR might be disadvantaged within largely disciplinary models of research assessment. There is a widely held assumption that interdisciplinary collaboration is less rewarding than collaborations within a discipline in terms of publications, recognition and career advancement. Peer reviews characterised by disciplinary norms and practices continue to influence departmental decisions about admission, funding and degree requirements. Faculty pursuing interdisciplinary research may find it hard to publish and have publications or contributions recognized by disciplinary peers (Feller, 2006; Mansilla, Feller \& Gardner, 2006). Moreover, it has been argued that there are relatively few 


\section{Let's work together}

high status interdisciplinary journals preventing scholars from pursuing IDR (Jacobs \& Frickel, 2009).

The peer process also applies to the assessment of funding applications and career appraisal. Even though research funders encourage interdisciplinary research, the evaluation and appraisal process represents another hurdle (Mansilla, et al., 2006). Assessments are often done by disciplinary experts using evaluation criteria they are familiar with. Workable quality criteria for the appraisal of interdisciplinary work have not yet been formalised (Jacobs \& Frickel, 2009; Klein, 2008), and identification of assessment criteria and the forms of expertise necessary for quality assessment is a major step in the advance of IDR.

This lack of epistemic clarity is likely to impact the organisation of IDR and the career trajectories of interdisciplinary researchers. In particular younger faculty and graduate students might be affected, since they generally express more enthusiasm for IDR than their more senior colleagues (Jacobs \& Frickel, 2009). If they cannot find a mentor or advisor who is engaged in IDR and who can help them to plan their career development, they will feel isolated, discouraged or misunderstood. They have to build up a publication and research record and demonstrate expertise within a discipline to be hired, promoted or to establish themselves in their careers. If they do not see any benefit for their own careers they might turn away from IDR.

How to promote individual motivation to engage in IDR? Given the multiple pressures on academic's time, it is of no surprise that some might argue that learning another field of knowledge detracts energy and time from their own discipline and that their career will be compromised. For others a driving motivational factor might be interest of learning about a different discipline because they came across a specific research topic that they cannot tackle without theoretical or methodological input from other domains. For IDR to succeed, appreciation, understanding, and respect for other disciplines must be developed. 


\section{Let's work together}

Both dialogue and common ground for theoretical and methodological debate must be established and maintained. Interdisciplinary teamwork also requires trust in another's skills and expertise and as well as mutual respect for other team members. Thus, a key challenge is to build supportive networks of students and faculty members, fostering IDR through institutional change.

Institutional structures. Current academic structuring has been identified by a number of authors as a key stumbling block for inter-departmental or cross-disciplinary cooperation (Feller, 2002, 2006; Jacobs \& Frickel, 2009; National Academy of Sciences, 2005). Funds are generally allocated to units or departments which in many cases are based on disciplines. The department and/or the discipline are generally the major site of student socialisation (Mansilla \& Gardner, 2006). Furthermore, buildings or floors of buildings often physically demarcate the departmental division of knowledge. Faculty are hired and promoted in this distinct units, which do not encourage interdepartmental or crossdisciplinary cooperation, but rather are associated with fights over allocations of resources (Sa, 2007). In the competition for scarce resources, interdisciplinary programs are often marginalized by some mainstream disciplines, or are assimilated by them. Moreover, evaluation, promotion and tenure processes often do not properly appraise collaborative and interdisciplinary work. The cultures and climate of departments and campuses are often indifferent or even hostile to such activities. Indeed, opponents of IDR have argued that interdisciplinarity is impossible to do (Fish, 1991).

To improve the recognition and reward structures for IDR it is vital to instigate change through the creation of supportive organisational structures. IDR can be promoted through a variety of means, ranging from the establishment of interdepartmental or interdisciplinary degree programs or organized research units (Sa, 2008). These research units can be created in different ways. Sometimes they are formed as a result of large external 


\section{Let's work together}

funding, through strategic administrative decisions, or through researcher led initiatives. A crucial concern is how to become financially sustainable after initial funding has stopped, and how to shift towards institutionalisation, i.e. the embedding of interdisciplinary programs into an organisations' structure (Borrego, Boden \& Newswander, 2014). In some cases academic institutions seek to institutionalise interdisciplinary research and teaching through mergers or restructuring of departments. In such cases, a healthy balance has to be struck between guidance from above, i.e. from the head of department, faculty dean or senior manager, and input from staff members through a bottom-up process and organic development of research interests. Academics typically respond best if they have some freedom to develop their research agenda as well as their immediate institutional context. Unless staff are experiencing some ownership of a changing context there will be problems and possibly resistance. Establishing IDR as a knowledge field has to be a collective effort, involving the building of bridges with firm foundations within disciplines.

\section{Conclusion}

Working together on societal problems can stimulate the process of creating new levels of understanding and provides opportunities for engaging in meaningful debate about theory, methodology and technique. The process of promoting and establishing IDR is not instantaneous, it will not always be smooth and involves working through conflicts and uncertainties. Yet, working together enables a move towards a more comprehensive understanding of how behaviours are related and how systems operate, providing new leverage of how to respond to complex questions that transcend specific disciplines. 


\section{Let's work together}

\section{References}

Borrego, M., Boden, D., \& Newswander, L. K. (2014). Sustained Change: Institutionalizing Interdisciplinary Graduate Education. Journal of Higher Education, 85(6), 858-885.

Feller, I. (2002). New organizations, old cultures: Strategy and implementation of interdisciplinary programs. Research Evaluation, 11, 109-116.

Feller, I. (2006). Multiple actors, multiple settings, multiple criteria: Issues in assessing interdisciplinary research. Research Evaluation, 15, 5-15.

Fish, S. (1991). Being interdisciplinary is so very hard to do. Issues in Integrative Studies, 9, $97-125$

Frodeman, R., Klein, JT. \& Mitcham, C. (Eds.) (2009). The Oxford Handbook of Interdisciplinarity. Oxford: Oxford University Press

Henry, S. (2005). Disciplinary hegemony meets interdisciplinary ascendancy: Can interdisciplinary/integrative studies survive, and if so, how? Issues in Integrative Studies, 23, 1-37.

Jacobs, J. A., \& Frickel, S. (2009). Interdisciplinarity: A Critical Assessment. Annual Review of Sociology (Vol. 35, pp. 43-65).

Jeffrey, P. (2003). Smoothing the waters: Observations on the process of cross-disciplinary research collaboration. Social Studies of Science, 33, 539-562.

Klein, J. (1990). Interdisciplinarity: History, theory, and practice. Detroit, MI: Wayne State University Press.

Klein, J. T. (2008). Evaluation of interdisciplinary and transdisciplinary research - A literature review. American Journal of Preventive Medicine, 35(2), S116-S123. 


\section{Let's work together}

Levitt, J.M and Thelwall, M. (2008). Is multidisciplinary research more highly cited? A macrolevel study. Journal of the American Society for Information Science and Technology 59(12):1973-1984.

Mansilla, V., Feller, I., \& Gardner, H. (2006). Quality assessments in interdisciplinary research and education. Research Evaluation, 15, 69-74.

Morillo F, Bordons M, Gomez I. (2003). Interdisciplinarity in Science: A Tentative Typology of Disciplines and Research Areas. Journal of the American Society for Information Science and Technology, 54:1237-49.

National Academy of Sciences. 2005. Facilitating Interdisciplinary Research. Washington DC: The National Academies Press.

OECD (1998). Interdisciplinarity in Science and Technology. Paris: OECD.

Repko, A.F. (2008). Interdisciplinary research. Process and theory. London: Sage

Sa, C.M. (2007). Planning for interdisciplinary research. Planning for Higher Education, $35(2), 18-28$.

Sa, C. M. (2008). 'Interdisciplinary strategies' in US research universities. Higher Education, $55(5), 537-552$. 